\title{
Towards the continuum limit of the critical endline of finite temperature QCD
}

\section{Yoshifumi Nakamura* ${ }^{* a, b}$, Xiao-Yong $\operatorname{Jin}^{c}$, Yoshinobu Kuramashi ${ }^{d, e, a}$, Shinji Takeda ${ }^{f, a}$ and Akira Ukawa ${ }^{a}$}

${ }^{a}$ RIKEN Advanced Institute for Computational Science,

Kobe, Hyogo 650-0047, Japan

${ }^{b}$ Graduate School of System Informatics, Department of Computational Sciences,

Kobe University,

Kobe, Hyogo 657-8501, Japan

${ }^{c}$ Argonne Leadership Computing Facility,

Argonne National Laboratory,

Argonne, IL 60439, USA

${ }^{d}$ Faculty of Pure and Applied Sciences,

University of Tsukuba,

Tsukuba, Ibaraki 305-8571, Japan

${ }^{e}$ Center for Computational Sciences,

University of Tsukuba,

Tsukuba, Ibaraki 305-8577, Japan

${ }^{f}$ Institute of Physics,

Kanazawa University,

Kanazawa 920-1192, Japan

${ }^{\dagger}$ Email: nakamuraeriken.jp

We presented "Critical endpoint of finite temperature phase transition for three flavor QCD", results for the critical endpoint of finite temperature phase transition of $N_{f}=3$ QCD at zero chemical potential. We employ the renormalization-group improved Iwasaki gauge action and non-perturbatively $O(a)$-improved Wilson-clover fermion action. The critical endpoint is determined by using the intersection point of kurtosis for the temporal size $N_{t}=4,6,8$. Additionally, we presented preliminary results at $N_{t}=10$ for the continuum limit of the critical endpoint.

The 33rd International Symposium on Lattice Field Theory

14 -18 July 2015

Kobe International Conference Center, Kobe, Japan

\footnotetext{
* Speaker.
} 


\section{Introduction}

We presented "Critical endpoint of finite temperature phase transition for three flavor QCD"[W], results for the critical endpoint of finite temperature phase transition of $N_{f}=3$ QCD at zero chemical potential at the temporal size $N_{t}=4,6,8$. Additionally, we presented preliminary results at $N_{t}=10$ for the continuum limit of the critical endpoint.

As we mentioned in Ref. [四, "Knowledge of QCD phase structure is the basis for understanding the physics of the strong interactions at finite temperature and density. Since simulations with finite chemical potential is plagued with the sign problem, it is important to clearly understand the finite temperature phase diagram as a function of light $\mathrm{u}$-d quark masses and strange quark mass before starting extensive studies at finite chemical potential. Analytical arguments indicate that the finite temperature transition with 3 massless quarks is of first order [D], which should then extend into the region of finite quark masses, ending at a line of critical points belonging to the $\mathrm{Z}(2)$ universality class [B]. Theoretical considerations alone cannot tell if the physical point with $m_{u d} / m_{s} \approx 25$ lies on the crossover side or the first order side of the critical line. The results of simulations done to date to locate the critical line are rather confusing. All results with staggered fermion action are consistent with the physical point being in the crossover region $[\because, \square, \square, \square, \mathbb{Q}, \mathbb{Q}, \mathbf{Q}]$. However, on the location of the critical line, while the standard staggered action yields the critical quark mass as

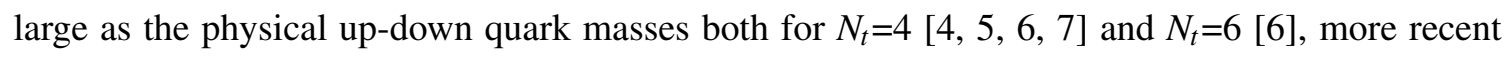
studies with improved staggered actions indicate that the critical quark mass is significantly smaller than the physical up-down quark masses [ $[\mathbb{0}, \mathbf{Q}]$. In fact improved staggered actions have yet not found first order signals. On the other hand, with the Wilson quark action, an extensive pioneering study with naive Wilson action and $N_{t}=4$ temporal lattice size found that the physical point lies in the first order phase transition region [ए0]. To clarify the issue, we study the critical endpoint for $N_{f}=3$ lattice QCD with non-perturbatively $O(a)$-improved Wilson fermion action, and attempt to estimate the continuum limit. Wilson type fermion action has exact flavor symmetry. In contrast, taste breaking with staggered type fermion action becomes large at coarse lattice spacings [Ш]]. We consider this to be a potentially more serious issue in comparison to lack of chiral symmetry with Wilson type fermion actions. Indeed the lack of chiral symmetry should not be a major problem for studying the critical endpoint which is expected to take place at a finite value of the quark mass."

For details of our method to determin the critical endpoint by using intersection point of kurtosis, please refer to Ref. [四]. To set the physical scale, we use Wilson flow [ㅁ] with $1 / \sqrt{t_{0}}=$ $1.347(30) \mathrm{GeV}$ [[13] (see also [ㅍ] ).

\section{Preliminary results at $N_{t}=10$}

We generate $\mathrm{O}(10,000)$ trajectories for each ensemble. We choose spatial lattice size $N_{l}=$ $16,20,24$ and cover the range $\kappa=0.14000-0.14013$ at $\beta=1.76, \kappa=0.13980-0.13990$ at $\beta=$ $1.77, \kappa=0.13950-0.13965$ at $\beta=1.78, \kappa=0.13925-0.13945$ at $\beta=1.79, \kappa=0.13875-0.13925$ at $\beta=1.80$. We compute plaquette, chiral condensate, their susceptibility, skewness and kurtosis. In Figs. $\mathbb{\square} \square$ we present results for susceptibility and kurtosis for plaquette and chiral condensate.

We find transition points in the simulated range at each $\beta$ from plaquette and chiral susceptibilities. Kurtosis is minimum at transition point at each $\beta$ and the value of minimum kurtosis gets 


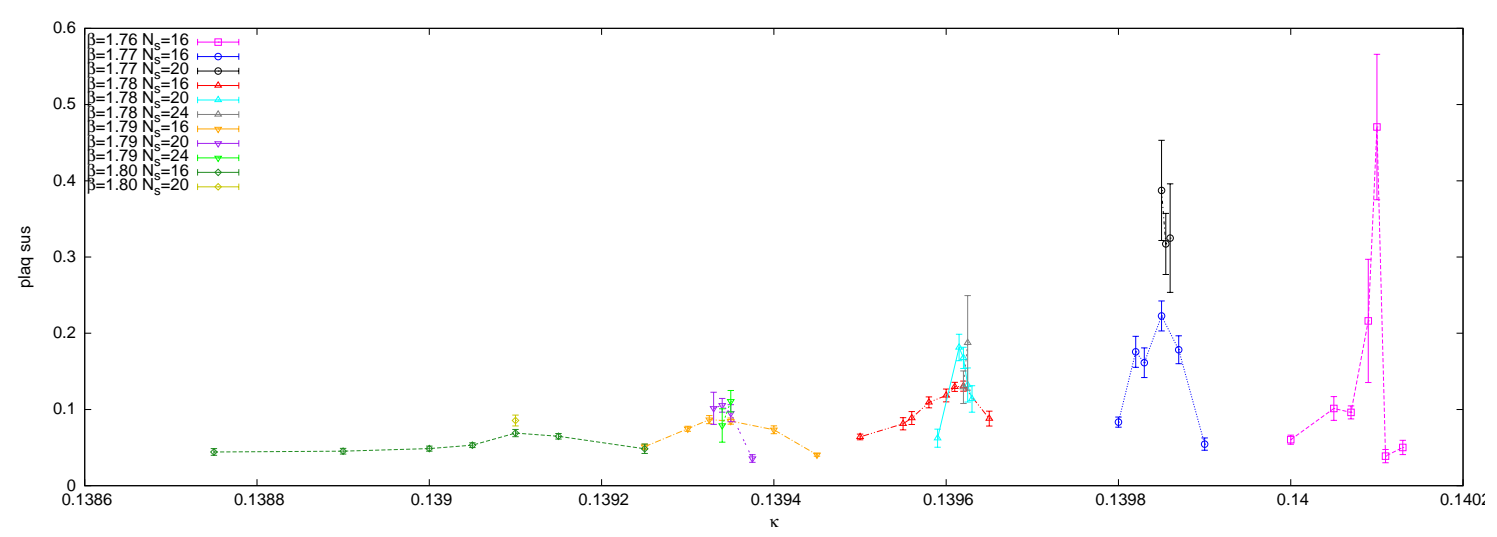

Figure 1: Susceptibility of plaquette as functions of $\kappa$ at $N_{t}=10$ for $\beta=1.76,1,77,1.78,1.79,1.80$.

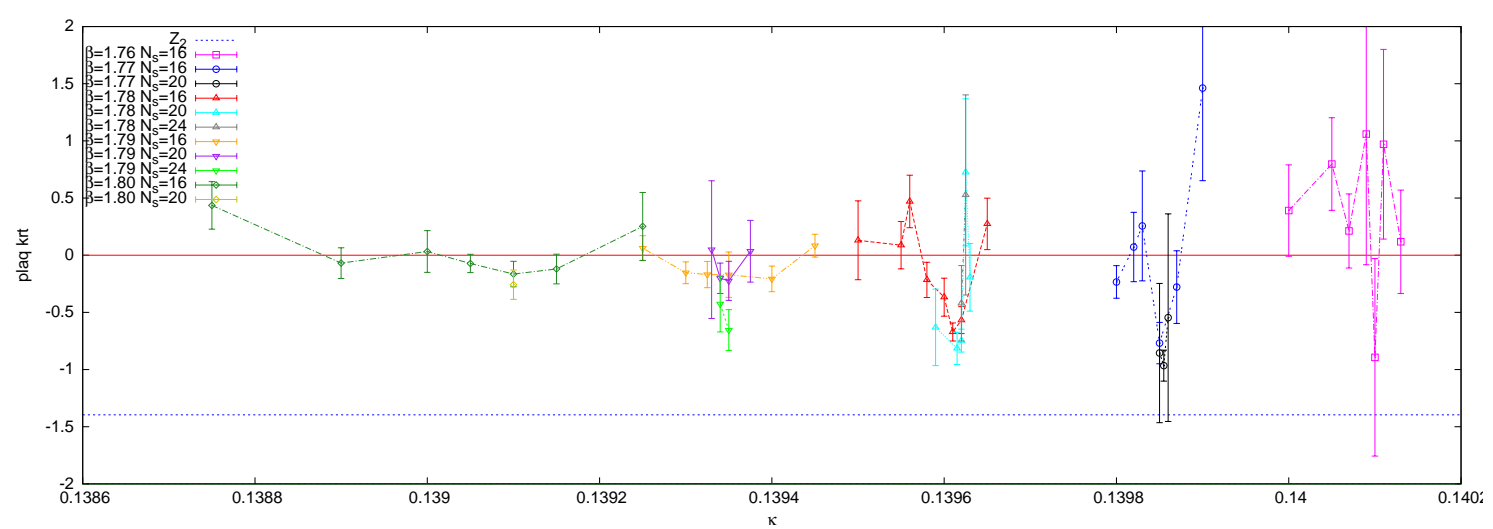

Figure 2: Kurtosis of plaquette as functions of $\kappa$ at $N_{t}=10$ for $\beta=1.76,1,77,1.78,1.79,1.80$.

smaller as decreasing $\beta$. These behaviors have been seen in our previous study. Unfortunately, we can not determine the endpoint of $\beta, \beta_{\mathrm{E}}$ because the volume dependence for minimum kurtosis is not clear due to lack of statistics. So, at the moment, we assume $\beta_{\mathrm{E}}=1.78(1)$ and try to look at how results at $N_{t}=10$ will affect the continuum extrapolation determined by using $N_{t}=6,8$ results.

In Fig. [1, we plot the determination of the pseudo scalar meson mass $m_{\mathrm{PS}, \mathrm{E}}$ and the transition temperature $T_{\mathrm{E}}$ at the critical endpoint in units of the Wilson flow scale $\sqrt{t_{0}} . T_{\mathrm{E}}$ depends on $\beta_{\mathrm{E}}$, while $m_{\mathrm{PS}, \mathrm{E}}$ does not very much around $\beta=1.78$. Fig. 6 shows the continuum extrapolation of $m_{\mathrm{PS}, \mathrm{E}}, T_{\mathrm{E}}$ obtained at $N_{t}=6,8$. If the assumption $\beta_{\mathrm{E}}=1.78(1)$ at $N_{t}=10$ is correct, $T_{\mathrm{E}}$ in the continuum limit would not change very much and $m_{\mathrm{PS}, \mathrm{E}}$ in the continuum limit may become smaller than results obtained by smaller $N_{t}$. 


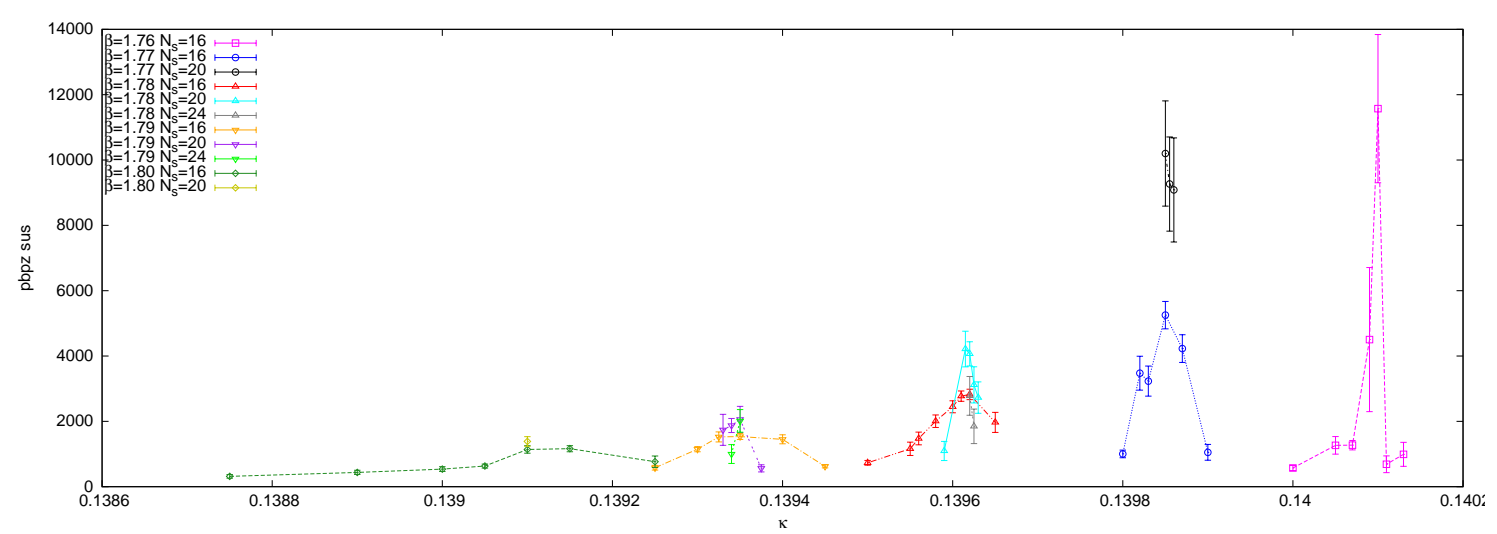

Figure 3: Susceptibility of chiral condensate as functions of $\kappa$ at $N_{t}=10$ for $\beta=1.76,1,77,1.78,1.79,1.80$.

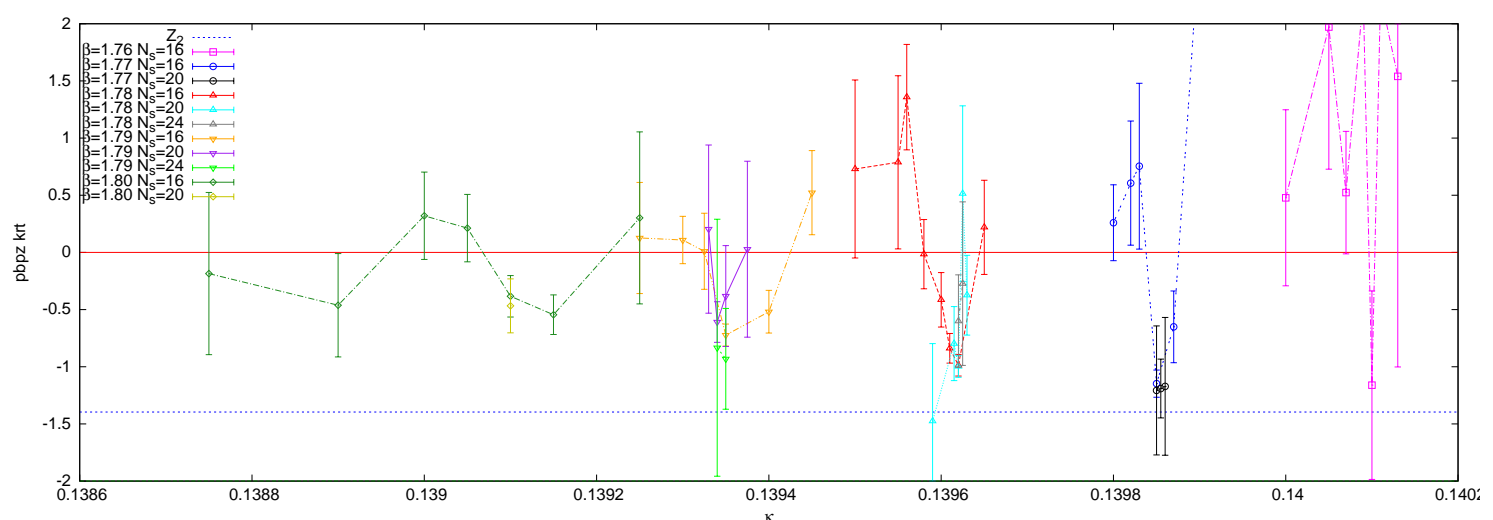

Figure 4: Kurtosis of chiral condensate as functions of $\kappa$ at $N_{t}=10$ for $\beta=1.76,1,77,1.78,1.79,1.80$.

\section{Summary}

We have investigated the critical endpoint of QCD with clover fermions and determined the critical endpoint by using the intersection points of the Binder cumulants and extrapolated to the continuum limit. If we assume $\beta_{\mathrm{E}}=1.78(1)$ at $N_{t}=10$, we find $T_{\mathrm{E}}$ in the continuum limit would not change very much, $m_{\mathrm{PS}, \mathrm{E}}$ in the continuum limit may become smaller than results at smaller $N_{t}$. We are doing further studies with high statistics at larger temporal sizes to obtain conclusive results.

\section{Acknowledgments}

The BQCD code [45] was used in this work. This research used computational resources of HA-PACS and COMA provided by Interdisciplinary Computational Science Program in Center for Computational Sciences, University of Tsukuba, System E at Kyoto University through the 


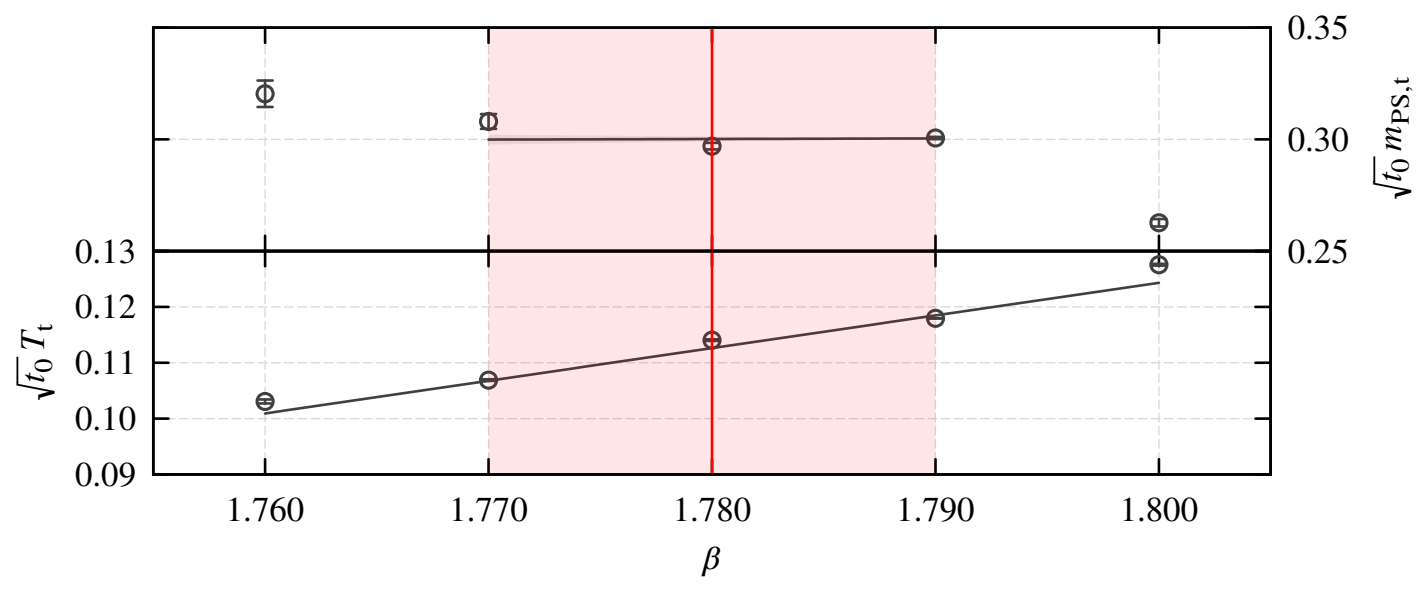

Figure 5: $\sqrt{t_{0}} m_{\mathrm{PS}, \mathrm{t}}, \sqrt{t_{0}} T_{\mathrm{t}}$ v.s. $\beta$ at $N_{t}=10$ with $\beta_{\mathrm{E}}$ and its one sigma error band when $\beta_{\mathrm{E}}=1.78(1)$ is assumed.
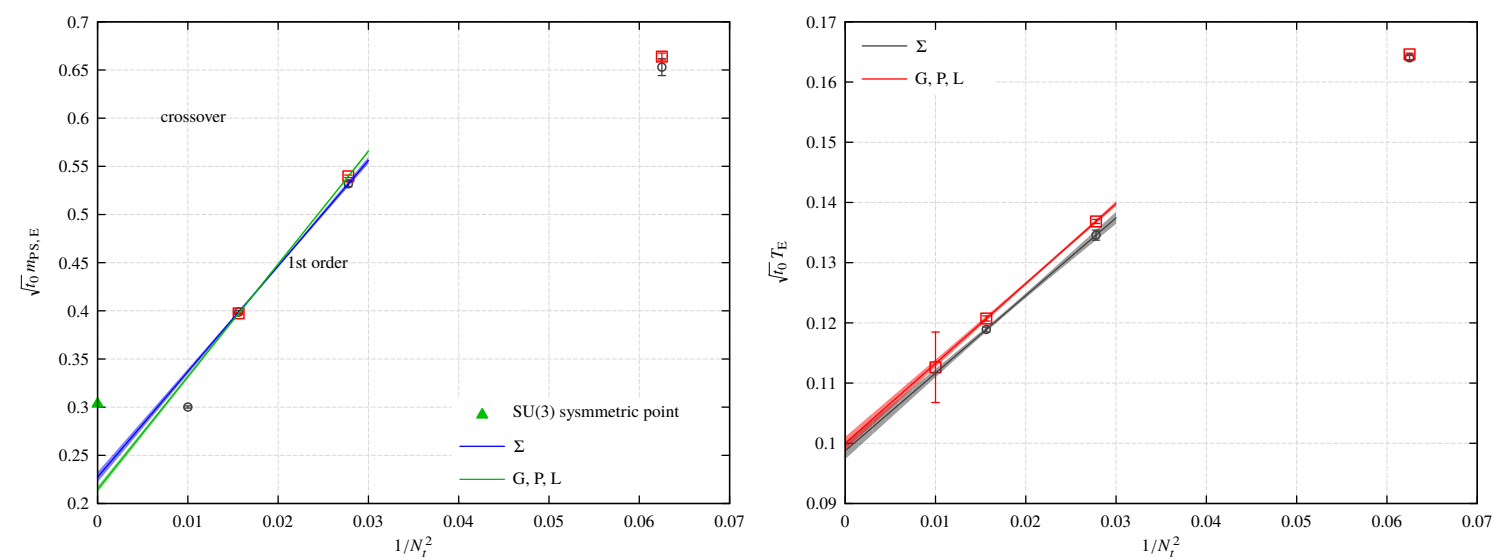

Figure 6: Continuum extrapolation of $\sqrt{t_{0}} m_{\mathrm{PS}, \mathrm{E}}$ and $\sqrt{t_{0}} T_{\mathrm{E}}$ as a function of $1 / N_{t}^{2}$ by using $N_{t}=6,8$. Results at $N_{t}=10$ are obtained by assuming $\beta_{\mathrm{E}}=1.78(1)$.

HPCI System Research project (Project ID:hp140180) and PRIMERGY CX400 tatara at Kyushu University. This work is supported by JSPS KAKENHI Grant Numbers 23740177 and 26800130. This work was supported by FOCUS Establishing Supercomputing Center of Excellence. 


\section{References}

[1] X.-Y. Jin et al., Phys. Rev. D 91, 014508 (2015), [arXiv:1411.7461 [hep-lat]].

[2] R. D. Pisarski and F. Wilczek, Phys. Rev. D 29, 338 (1984).

[3] S. Gavin, A. Gocksch and R. D. Pisarski, Phys. Rev. D 49, 3079 (1994).

[4] S. Aoki et al. (JLQCD Collaboration), Nucl. Phys. Proc.Suppl. 73, 459 (1999).

[5] F. Karsch, E. Laermann and C. Schmidt, Phys. Lett. B 52041 (2001), [arXiv:hep-lat/0107020].

[6] P. de Forcrand and O. Philipsen, JHEP 0701, 077 (2007), [arXiv:hep-lat/0607017].

[7] D. Smith and C. Schmidt, PoS(Lattice 2011), 216 (2011). [arXiv:1109.6729[hep-lat]]

[8] G. Endrődi et al., PoS(Lattice 2007), 182 (2007), [arXiv:0710.0998 [hep-lat]].

[9] H.-T. Ding et al., PoS(Lattice 2011), 191 (2011), [arXiv:1111.0185 [hep-lat]].

[10] Y. Iwasaki et al., Phys. Rev. D 54, 7010 (1996), [arXiv:hep-lat/9605030]

[11] A. Bazavov et al., Phys. Rev. D 85, 054503 (2012), [ arXiv:1111.1710 [hep-lat]]

[12] M. Lüscher, JHEP 1008, 071 (2010), [ arXiv:1006.4518 [hep-lat]].

[13] S. Borsanyi et al., JHEP 1209, 010 (2012), [arXiv:1203.4469 [hep-lat]]

[14] R. Horsley et al., PoS(Lattice 2013), 249 (2013), [ arXiv:1311.5010 [hep-lat]].

[15] Y. Nakamura and H. Stüben, PoS(Lattice 2010), 040 (2010), [arXiv:1011.0199 [hep-lat]]. 